\title{
Context Weaver: Awareness and Feedback in Networked Mobile Crowdsourcing Tools
}

\author{
Tomoyo Sasao ${ }^{\mathrm{a}}$, Shin'ichi Konomi ${ }^{\mathrm{b}, *}$, Masatoshi Arikawa $^{\mathrm{b}}$, Hideyuki Fujita ${ }^{\mathrm{c}}$ \\ ${ }^{a}$ Graduate School of Frontier Sciences, the University of Tokyo, Kashiwa, Japan \\ ${ }^{b}$ Center for Spatial Information Science, the University of Tokyo, Kashiwa, Japan \\ ${ }^{c}$ Graduate School of Information Systems, University of Electro-Communications, Tokyo, \\ Japan
}

\begin{abstract}
Mobile crowdsourcing allows people to collect data using a large pool of participants. In this paper, we focus on mobile crowdsourcing for citizens to solve local issues in context. We argue that such crowdsourcing environments need to support exploration, a continuous, opportunistic, and multi-perspective process that existing crowd sensing systems cannot easily support. We have developed a system called Context Weaver, which connect participants using networked mobile devices in order to support collaborative exploration, and conducted field trials to understand the effect of networking participants in the crowdsourced data-collection activities that encompass planning, execution, and analysis phases. We discuss a methodology for exploratory mobile crowdsourcing by citizens based on the provision of mutual awareness and rapid feedback in context. The proposed methodology can provide a basis for a model of networked mobile crowdsourcing which can exploit not only the man-power but also the creativity of citizens to gather relevant data.
\end{abstract}

\section{Introduction}

Mobile crowdsourcing allows people to collect data using a large pool of participants who move around in public spaces. From the perspectives of participants, mobile crowdsourcing can be understood as a novel form of mobile 5 work, including the types of work that can contribute to local communities and civic societies. Thus, one might argue that mobile crowdsourcing can provide opportunities of citizen participation on the move. Citizen participation

\footnotetext{
*Corresponding author

Email address: konomi@csis.u-tokyo.ac.jp (Shin'ichi Konomi)
}

Preprint submitted to Special Issue on "Crowdsourcing" in Computer Networks Journal February 26, 2015

(C) 2015. This manuscript version is made available under the Elsevier user license http://www.elsevier.com/open-access/userlicense/1.0/ 
plays a key role in the design of the spaces we inhabit as well as the design of the solutions to the problems we face. The ubiquity of smartphones and tablet computers enables various kinds of mobile crowdsourcing methods for citizen participation, including participatory sensing and citizen science. They seem to be on the verge of making a significant impact in the real world, as people increasingly use pervasive technologies to share information about local problems (e.g., FixMyStreet [1]), geo-tagged radiation Geiger counter readings 15 (e.g., Japan Geigermap), and so on. One may describe such location-specific crowdsourcing as geocentric [2] since each geographic space is a shared focus of participants.

A key limitation to these existing technologies for citizen participation is the relative lack of the support for exploratory data collection. This limitation could trivialize the impact of mobile crowdsourcing in urban spaces since many of the real-world problems in a city can be considered ill-structured, 'wicked' problems [3] that one cannot simply "first understand, then solve." Coping with such problems can require a more open-ended, exploratory data collection environment based on situated actions [4], which considers different perspectives of participants and supports continuous and opportunistic processes.

In this context, we propose a geocentric mobile crowdsourcing approach that connects mobile peers, thereby creating a network of co-located workers through mutual sharing and awareness. In doing so, we exploit social tagging, which enables rapid exploration in Web search and possibly provides a kind of scaffolding 30 for learning as well [5]. We extend and integrate such tag-based approach to integrate data collection and its social context. We also introduce workflows to provide an integrated support for the work processes that contextualize data collection. We propose Context Weaver, which is a mobile data collection environment that supports not only data collection but also social and work-related

35 contexts of data collection, with the aim to facilitate exploration in geocentric mobile crowdsourcing.

The Context Weaver environment consists of a pair of complementary tools: one is a tag-based mobile tool for supporting participatory fieldwork, and the other is a collaborative shared 'tabletop' tool to support collaborative analy-

40 sis and discussion in the field. A tabletop can support impromptu face-to-face collaboration, and could allow for quick and effective visual analytics tasks [6]. Through the field trials of these co-located mobile collaboration tools, we discuss a networking methodology for supporting exploration in mobile geocentric crowdsourcing environments for citizen participation as well as the implications 45 of mutual awareness and the tradeoff of rapid in situ feedback and ease of par- 
ticipation. The networking encompasses the planning and analysis processes as well as the data collection process itself.

\section{Related Works}

\subsection{Mobile Crowdsourcing and Crowd Sensing}

Participatory data collection and sharing environments have been used for the support of local communities. FixMyStreet is a website through which people can report and share information about local issues such as potholes and broken streetlights [1]. It has accompanying smartphone apps as well as RSS/email alert feature that allows users to be notified of incoming reports based on a postcode or street name and area. The Ushahidi platform supports collection, mapping, and sharing of data from crowds via text messages, email, twitter, and web, and has been used to facilitate crisis mapping [7]. In addition, researchers have developed urban sensing platforms [8] as well as participatory sensing and citizen science tools to monitor air quality, watersheds, local parks,

60 university campuses, safety in a city $[9,10,11,12$, 13], etc. Alt et al. propose a location-aware mobile crowdsourcing platform in which requesters upload tasks to a database and workers retrieve the tasks related to a given location using mobile phones [14].

\subsection{Collaboration in Crowdsourcing}

In crowdsourcing, collaborative functions help crowds to complete not only simple tasks requiring little time and effort but also complex and interdependent tasks that require coordination among workers. We have identified the following three approaches to supporting complex crowdsourcing tasks. Although not an exhaustive list, it has informed the design of our system. The list has some resemblance with the crowdsourcing dimensions proposed by Pan and Blevis [15], although we focus more on mechanisms for facilitating collaboration than the analysis of crowdsourcing practices in general.

Workflow When the task is too large for one person or can be done better through collaborative work, one might need appropriate workflows that guide crowds and allow them to take charge of subtasks and work cooperatively. CrowdForge is one of the web-based crowdsourcing prototypes that support wide-ranging complex problems by breaking them down into sequences of simpler subtasks based on general subtask types (i.e., partition, maps, reduces)[16]. Their case studies show that the workflow-based approach leads to better quality of the results than conventional approach 
without collaboration. However, one of the limitations of the workflowbased approaches is that some tasks cannot be easily decomposed into independent subtasks, and coordination of workers can sometimes be difficult. Another research that exploits collective intelligence of groups in complex work scenarios discusses the research value chain [17] that involves a specific type of workflows based on research procedures. Although these are designed for web-based crowdsourcing, similar approaches could potentially be exploited for mobile crowdsourcing.

Guidance Kittur and Kraut discuss that even a free and open environment such as Wikipedia can be benefitted from authoritative management [18]. Individuals, groups or organization who can guide the work of participants could play important roles in similar complex crowd work environments. They can influence participants in different ways ranging from authoritarian supervision to democratic facilitation. Their guidance can be directly communicated to participants or embedded in the work structures that they introduce to participants.

Coordination support There are two types of coordination among workers in formative steps of collaborative work [18]. As cited in [18], one is explicit coordination based on direct communication and verbal planning and the other is implicit coordination based on workgroup structure, unspoken expectations and shared mental models of the task to be accomplished. Both types of coordination could enrich the "wisdom of the crowd" and improve the quality of their work but they also introduce the risk of conflicts among workers. Therefore, coordination functions in crowdsourcing environments should be designed carefully.

\subsection{Collaboration Support in Mobile Crowdsourcing}

Väätäjä et al. [19] focus on a co-creating process for news articles, which involves the coordination among editorial staff and mobile journalists. Their workflow consists of the 5 steps that are based on traditional practices and each step needs different skills. Their case studies show that such an approach worked for small and simple assignments, whereas it did not work well for larger complex assignments due to "the lack of a possibility for discussing the topic" with mobile journalists. That is, complex mobile crowdsourcing tasks would require effective communication among participants.

In traditional urban design and management, citizens participation is known to be critical at the initial stages that involve exploration of local issues and so-

lutions. If crowdsourcing systems can support citizens' collaboration during the 
task formulation stage, mobile crowdsourcing could support a wider range of applications for the support of local and social issues. Relevant to this dis${ }_{120}$ cussion is mobile tools that support exploratory data collection. For example, GeoTagger [20] is a participatory environmental inquiry system based on citizen science. The system's purpose is to encourage children to explore their environment, connect with peers, and have conversations about observed data. It allows users to create and share tags along with comments during fieldwork although they do not consider workflows. NatureNet [21] focuses on co-designing mobile crowdsourcing environments with citizens and professional design teams. Their user studies show that their prototype has potential for generating interest and enthusiasm from citizens who participate in citizen science activities. Both GeoTagger and NatureNet aim to support complex work using mobile tools.

${ }_{130}$ However, they do not explicitly support structures and flows of mobile data collection work, whose patterns could be identified by examining conventional practices by citizens.

\section{Existing Practices}

\subsection{Participatory Mobile Data Collection}

To get a better sense of participatory data collection activities in the real world, we participated in data-gathering events that took place in Japanese cities, and looked into existing practices by groups of citizens [9]. These two preliminary case studies are revealing of the importance of collaborative exploration:

${ }_{140}$ Citizen Sensing after a Nuclear Disaster. The recent nuclear disaster in Fukushima caused fears and concerns of radiation hotspots in some Japanese cities, including the city of Kashiwa in Chiba. A grassroots organization collected radiation data at more than thirty parks in Kashiwa during the period between November 2011 and December 2012, using radiation sensors (Mr.Gamma 145 A2700). A close look at their fieldwork in February 2012 allowed us to understand the teamwork practices that had been shaped since the beginning of this collective endeavor. A team of three participants includes a measurer who operates a Mr.Gamma device, a recorder who records measurements and locations on a piece of paper, and an explorer who explores the 'suspicious' spots to determine the exact points that must be measured. Participants are opportunistic and adapt their work to different situations in the field, and the teams share their results and insights during lunchtime before embarking on afternoon fieldwork. These collaborative work practices provide a learning opportunity for 
novice participants, who eventually become more skillful in finding radiation

155

Participatory Fieldwork in Suburban Tokyo. A community fieldwork event was held in suburban Tokyo to discover and map attractive elements using maps and digital cameras. Four or five participants worked together in each team taking photographs, jotting down their findings and ideas, and recording the relevant planning session to discuss and agree on the kind of information the team wants to collect, (2) the fieldwork itself, and (3) a post-hoc discussion and map-making session. A facilitator and a graphic designer help the teams in step 1 and 3, respectively. Overall, it is more open-ended and exploratory than the first case, and characterized by the diversity and sharing of perspectives and expertise.

Mobile crowdsourcing can involve different degrees of exploration. In sensorbased mobile crowdsourcing environments, people may use a smartphone app that captures location information and sensor data automatically. All they have to do is to launch the app and put the smartphone in their pockets, requiring little conscious work. Other mobile crowdsourcing environments may require people to collect data more collaboratively such as the environments for recording radiation measurements and collecting information in participatory fieldwork.

\subsection{Exploratory Information Gathering}

To design a support environment for exploratory citizen sensing, we can learn from existing approaches and practices in different types of information work. Field observation by ethnographers can be classified into descriptive, focused, and selective observation [22]. Descriptive observation views the environment "through a wide-angle lens" to describe the environment thoroughly, which enables a high-level analysis to explore and identify important domains in the environment. Focused observation, then, uses the identified key domains to narrow down the scope of observation, and enables detailed analysis to provide a start point for deeper, selective observation, which is followed by further analysis. The whole process is exploratory in nature, and analytic work is intertwined with shifting observation work. It is a cyclic and iterative process, and analysis can create a need for additional data collection, which generates data for additional analysis, and so on.

A professional technology analyst, who gathers information, makes sense of it, and packages it as a reading material, needs to explore a mountain of 
information. Analysts have relatively well-defined goal, and they use specific concepts, or a knowledge schema, to collect and make sense of incoming information [23]. Their sensemaking is interleaved with data collection. This type of information-centric work can be performed in a collaborative manner as well. Social search systems, which emphasize that information seeking is embedded within social relationships, can provide useful cues that make exploration more efficient, and foster learning and investigation activities [5].

\section{The Context Weaver System}

Based on the analysis of the existing practices and our preliminary design exercise [24], we developed a prototype environment called Context Weaver, which supports recruited participants to collect data in exploratory manners. We have designed the key features of Context Weaver based on our informal observation of existing participatory mobile data collection practices, which are described in Section 3.1. Context Weaver considers three activities related to exploratory mobile crowdsourcing: (1) planning, (2) data collection and (3) analysis, which correspond to the structure of the participatory fieldwork we observed in suburban Tokyo.

Moreover, dynamic social tagging can support communication in complex mobile tasks such as exploratory social search. Tags can be used to describe and organize collected information. They can also be communicated for socialization, awareness, coordination, reflection, inspiration, and learning, which can be beneficial in mobile data collection practices that are similar to the cases we informally observed. For example, in the planning phase, individuals or groups can create tags such as 'runners' to plan a fieldwork that looks at healthy activities in a park. Then, during the fieldwork, a participant may notice that there are no shades near the entrance of the park and create a tag 'need shades for runners'. This tag can then be shared with other team members who eventually collect relevant geotagged photos. Afterwards, they can discuss issues and ideas based on the data collected in this manner.

As shown in Figure 1, Context Weaver supports quick iteration of planning, work and analysis in the field, which is different from conventional approaches that focus on data collection during fieldwork. In many citizen sensing projects, there is a significant time lag between data collection and analysis because people need to go back to a meeting room or a home to analyze and discuss the collected data. Context Weaver exploits tablet computers to enable quick rendezvousing of participants for collaborative data analysis in the field without the 


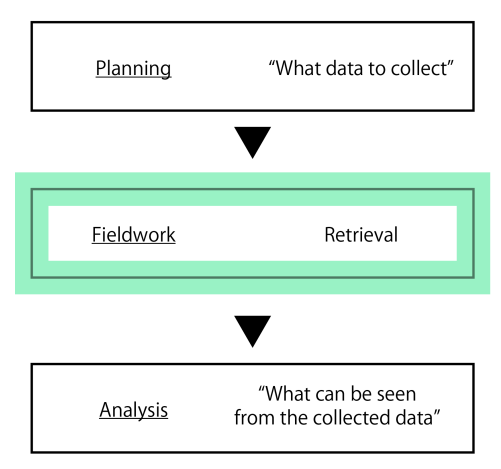

(a) Conventional Participatory Fieldwork

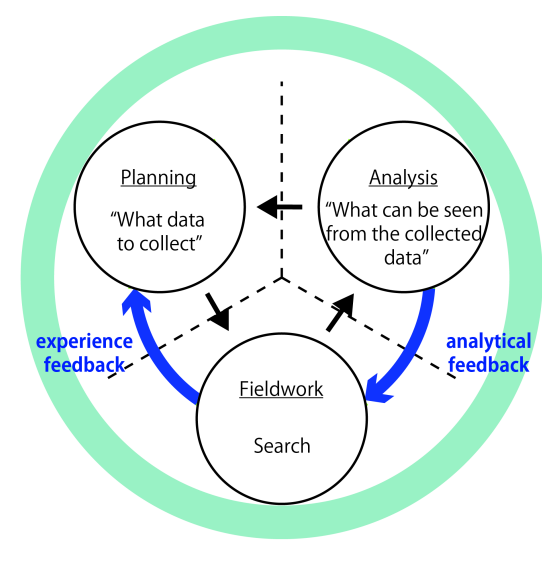

(b) Context Weaver

Figure 1: Comparison of Conventional Participatory Fieldwork and Context Weaver

undesirable time lag. It allows people to become aware of insufficient coverage in situ or to revise questions or strategies of data collection so that participants can work more effectively as a group. As shown in Figure 1b, our system also uses social tagging to provide experiential feedback and analytical feedback. Experiential feedback connects the fieldwork phase back to the planning phase in order to inform the planning process with participants' experiences in the field. This is done by allowing participants to create tags during fieldwork and sharing them at the planning phase of the next iteration. Analytical feedback connects the analysis phase back to the fieldwork phase in order to inform the fieldwork process with evolving analytical understandings. This is done by allowing participants to create tags during analysis and sharing them at the fieldwork phase of the next iteration. Again, all of these can take place quickly as participants can use tablets for in situ planning and analysis.

The Context Weaver system consists of two complementary tools: Scene Memo for mobile data collection and Discussion Table for collaborative analysis and discussion. As shown in Figure 2a, Scene Memo can be used to take pictures and add tags to the pictures easily on a smartphone (or a tablet computer). When the user takes a picture, Scene Memo immediately uploads the picture and the corresponding tags to a shared server. This way, the tags can be shared quickly for networked concurrent activities. Moreover, Scene Memo provides the functionality to order tags according to different criteria, based on relevant time, location, user names, activities, and the number of links and 'likes.' 
Scene Memo supports three modes of tag sharing (i.e., M1, M2, and M3), which allows for flexible integration of Scene Memo into the activities of re-

\subsection{Method}

As we focus on the design of mobile tools for carrying out data collection tasks, our work complements research projects that discuss participant recruitment processes [12] or task-participant matching [25, 26]. For the purpose of our 
(a) User Interface of Scene Memo

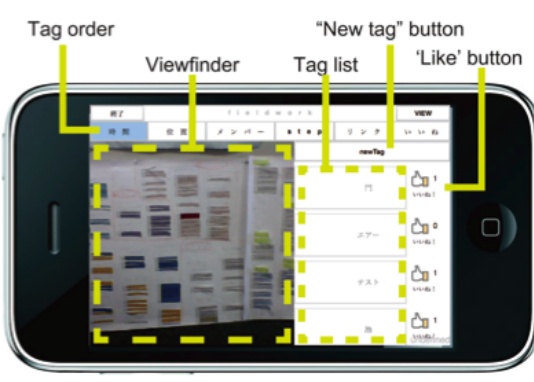

Look for things to record

Release shutter

Add tag to recorded data (photo)

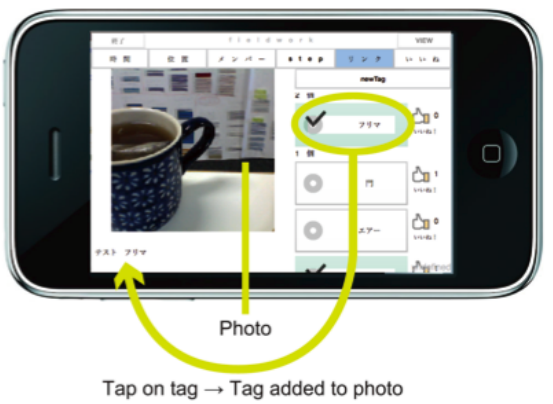

Tap on tag $\rightarrow$ Tag added to photo

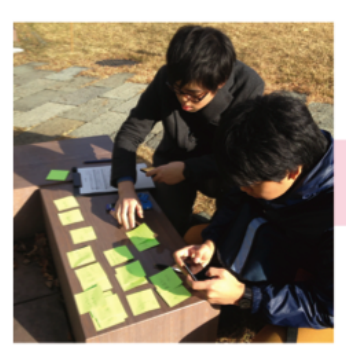

Planning

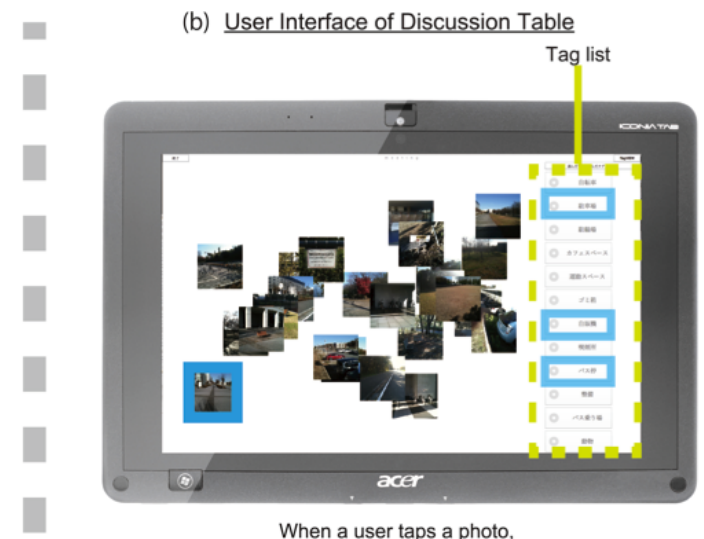

tags that have been attached to it are emphasized.

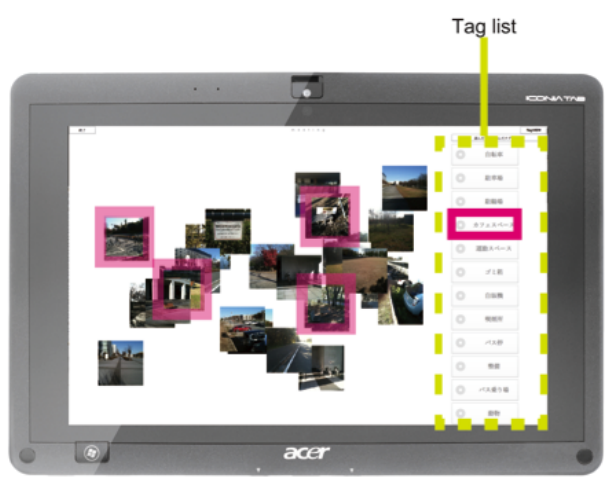

When a user taps a tag

photos that have been attached to it are emphasized.

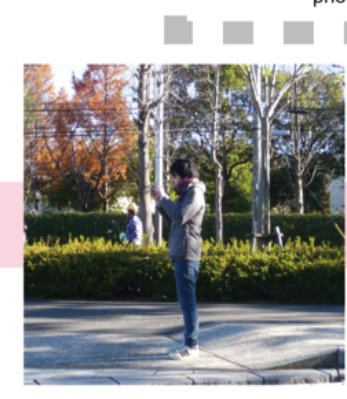

Fieldwork

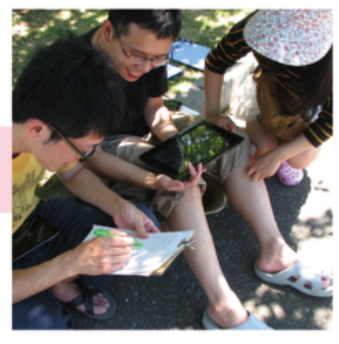

Analysis and Discussion

(c) Experiments

Figure 2: Context Weaver integrates Scene Memo and Discussion Table to support planning, fieldwork, and analysis/discussion 
methods (e.g., offline social networks and physical flyers at local venues), and asked them to use Context Weaver to collect data at three university campuses with the purpose of improving the campus environments (see Figure 2c). Four women and 11 men, aged 20-30, participated in this study. $73 \%$ of them had previous experience with fieldwork. We grouped participants to form 6 teams $(\mathrm{t} 1, \mathrm{t} 2, \cdots, \mathrm{t} 6)$, each having 2 or 3 members. Each team carried out the mobile data collection task twice under different conditions at one of the three university campuses. Participants were compensated with a prepaid shopping card with 1,000 Japanese Yen (approx. 10 US Dollars).

We prepared smartphones (iPhone 3GS) with the pre-installed Scene Memo application as well as a tablet computer (Acer ICONIA TAB W500) with the pre-installed Discussion Table application. These applications have the feature to display an up-to-date list of tags, which are synched with the shared server every 30 seconds. The tablet computer's screen size is 10.1 inch, which we expected would be large enough for touch-based interactions by a few people around the device. Participants received enough training to familialize themselves with the devices and applications.

Participants used the applications based on the following three steps. The first step is the planning of the activity in the field, which lasted for about 15 300 minutes. In this step, participants planned the data collection activities for improving university campus environments. Participants first discussed issues and ideas related to campus improvement and then decided on what information to collect, where to explore, and other details of the data collection activities. As the result of the planning step, keyword tags were generated so that participants can use them during the actual mobile data collection in the field. In order to examine the effect of shared tags, which we expect to serve as 'glues' to connect mobile workers, we asked participants to create tags under the following two conditions:

Planning Condition P0: Participants create private tags individually.

Planning Condition P1: Participants create shared tags together. The tags are shared with other team members.

The second step is mobile data collection in the field, in which participants explore the university campuses and collect data by taking photos and tagging the photos. This step lasted for about 20 minutes. In order to examine the effect 315 of connecting participants during this step, the tags created by participants during mobile collection activities were kept locally on mobile devices or shared with other participants in realtime via an online database server. : 
Fieldwork Condition F0: The tags that participants create during fieldwork are kept private on local devices.

320 Fieldwork Condition F1: The tags that participants create during fieldwork are shared with other team members in realtime via an online server.

Each team used our system twice under different planning and fieldwork conditions. We randomized the assignment of the teams and conditions to minimize the learning effect due to the experiment order. In addition, we divide each university campus into two zones so that participants can explore a different zone in each round.

The third step is analysis and discussion. In this step, participants analyze and discuss the collected data using a tablet device, and generate a list of ideas for improving university campus environments. At this stage, all participants can view all tags including the ones that are created privately in the planning and/or fieldwork steps. In other words, tags created under any of the planning and fieldwork conditions are visible to all team members. This step lasted for about 15 minutes.

After the third step, participants answered a questionnaire about the usability and usefulness of the system, followed by a 30-minute interview. The conversations during the first step, the third step, and the interviews are recorded and transcribed for qualitative analysis. These transcripts are coded and analyzed by two researchers based on grounded theory. The questionnaire includes detailed questions about the usage of tags. The system log files were used 340 to analyze participants' interactions with the applications along with relevant contextual information such as location and time. Additionally, we used NASATLX to measure the system's workload, which however is outside the scope of this paper.

\subsection{Result}

\section{5.2.1. Quantitative Results}

Participants rated the usefulness of tags using a likert scale from 1 to 5 , with 5 indicating useful, 4 somewhat useful, 3 neutral, 2 somewhat useless, and 1 useless. During fieldwork, the tags made in Step 1 (planning) were perceived to be more useful (mean: 4.15, SD: 0.38) than the tags made in Step 2 (fieldwork) (mean: 3.67, SD: 0.60). Similarly, during analysis and discussion, the tags made in Step 1 (planning) were perceived to be more useful (mean: 4.13, SD: 0.27) than the tags made in Step 2 (fieldwork) (mean: 3.78, SD: 0.63). Interestingly, when participants create tags together with other team members during the 
planning step (P1) and share the tags they create during fieldwork (F1), the

result is reversed: the tags created in Step 2(fieldwork) were perceived as more useful than the tags created in Step 1 (planning).

During the analysis phase, 22, 20, 15 and 16 ideas were generated fro P0F0, $\mathrm{P} 0 \mathrm{~F} 1, \mathrm{P} 1 \mathrm{~F} 0$ and P1F1, respectively. It appears that there are more ideas generated for $\mathrm{P} 0 \mathrm{~F} 0$ and $\mathrm{P} 0 \mathrm{~F} 1$, in which participants created private tags individually during the planning step. We will discuss the implication of this result along with the relevant qualitative results.

Figure 3 shows the comparison of the number of tag usage during fieldwork. The areas in blue indicate the tags created in the planning phase, and the areas in red indicate the ones created in the fieldwork phase. The labels (P0F0, P0F1, $\mathrm{P} 1 \mathrm{~F} 0$, and $\mathrm{P} 1 \mathrm{~F} 1$ ) indicate the combinations of the corresponding conditions. Overall, the tags created during the planning phase were used more than the ones created during the fieldwork phase.
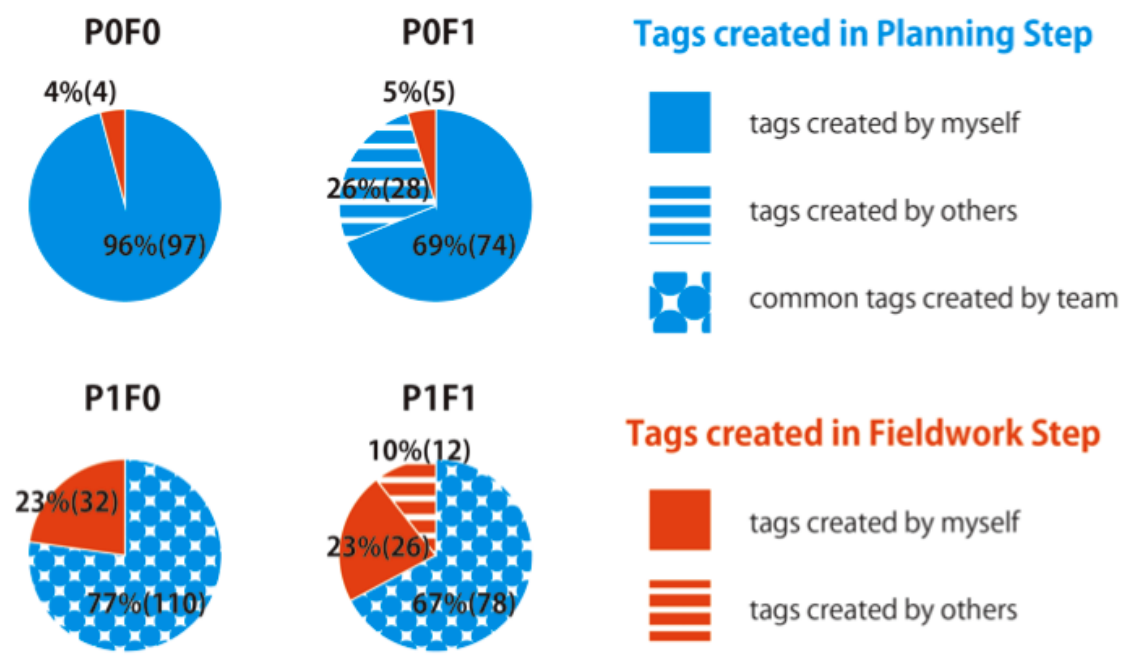

Figure 3: Comparison of the Number of Tag Usage during Fieldwork

The pie chart for $\mathrm{P} 0 \mathrm{~F} 0$ shows the number of tag usage when tags were private during the planning and fieldwork phases. Not surprisingly, the private tags from the planning step were used the most. The pie chart for P0F1 shows the number of tag usage when tags were private during the planning phase but they were shared during the fieldwork phase. Again, the private tags created 
during the planning phase were used most frequently. However, unlike P0F0, participants also used shared tags 26 percent of the time.

The pie chart for P1F0 shows the number of tag usage when tags were shared during the planning phase but they were private during fieldwork. In this case, the shared tags created during the planning phase were used most frequently, but participants used private tags created during fieldwork 23 percent of the time. The pie chart for P1F1 shows the number of tag usage when tags were shared during the planning and fieldwork phases. Again, the shared tags created during the planning phase were used most frequently. Interestingly, the tags that participants created in situ are most frequently used for the condition P1F1 as Figure 3 shows that participants used the shared or private tags created during fieldwork 33 percent of the time.

Moreover, our survey results show that $76 \%$ participants perceived the planning step meaningful or somewhat meaningful, whereas all participants perceived the analysis step meaningful or somewhat meaningful. The analysis step was rated the highest in terms of discovery and learning. 54\%, $79 \%$ and $97 \%$ participants thought they discovered something new during the planning, fieldwork and analysis steps, respectively. Also, 51\%, $64 \%$ and $84 \%$ participants thought they learned something during the planning, fieldwork and analysis steps, respectively.

Figure 4 shows the number and usefulness of the ideas generated in the analysis phase. More ideas were generated for conditions P0F0 and P0F1 than other conditions. Perceived usefulness of generated ideas seems higher in these conditions as well. In both of these conditions, participants created private tags freely during the planning step. We will discuss these results along with our qualitative findings, and suggest that our method with the right condition may increase the quantity and usefulness of ideas compared to conventional mobile crowdsourcing approaches in which goals are framed in advance.

Finally, we like to briefly mention that the NASA-TLX result suggests that creating and sharing tags in realtime during fieldwork (i.e., condition F1) could reduce the frustration related to the use of Scene Memo.

\subsubsection{Qualitative Results}

We now describe the results based on the qualitative interviews. Note that the quotes were translated from Japanese.

Conceptual scale. Participants noticed the difference of the tags created in the planning and fieldwork phases. For example, four participants talked about the 


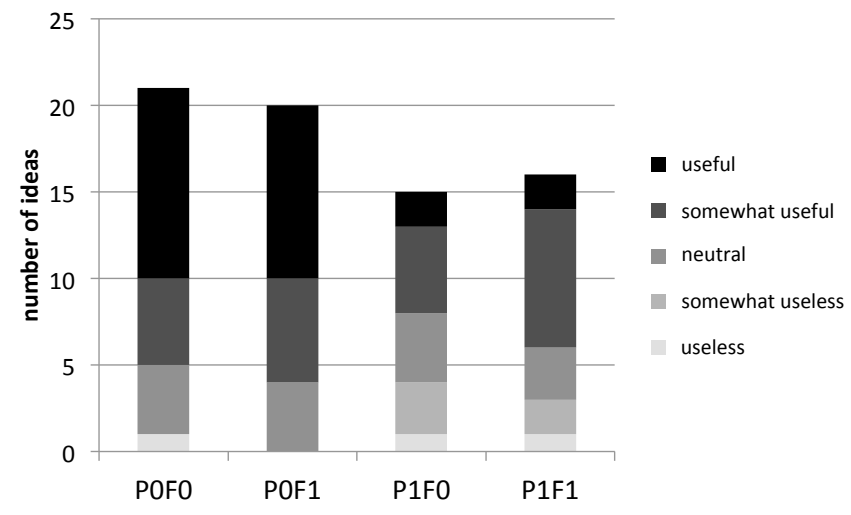

Figure 4: Quantity and Usefulness of Generated Ideas

difference of conceptual broadness (or narrowness) of the tags that were created in different ways at different times. Tags created in the planning phase were perceived as "conceptually broad" or "categorically broad." In contrast, tags created in the fieldwork phase were perceived as 'detailed,' "conceptually narrow" or "more zoomed-in information concepts." In addition, some participants said that they created conceptually broad tags when they were to be shared with others, and conceptually narrow tags when they were to be used personally. During the analysis phase, some teams first selected conceptually broad tags such as 'road' to take a look at various photos related to broad categories, and then selected conceptually narrow tags such as "pothole that affects bikers" to take a look at a small number of photos related to specific descriptions. These results suggest that tags' conceptual scale influences their use, and, conversely, the tags' intended use influences their conceptual scale.

Discovery. Four participants said that they understood different perspectives of other participants by taking a look at shared tags. They also mentioned that they used such understanding as an alternative lens to look at the field, and discovered new things. For example, one participant mentioned that seeing the tags "street lamp" and "narrow street" led to the discovery that "[streets are] indeed narrow" and "aha, a street lamp is on."

Learning. Two participants learned appropriate tagging methods for the things they wanted to record. They also learned from the ways other participants performed the work. For example, one of the participants said, "I was doing what 
I had never done before. It was pretty easy to go about the fieldwork because I could do it as I was looking at the ways others were doing it." Moreover, the analysis phase provided opportunities for learning and discovery. One of the participants commented on his experience during the analysis step, "In some way, I realized that she looks at things differently than me, yeah, I think there was something to learn." This is in line with our quantitative results that show $97 \%$ participants thought they discovered something new in the analysis phase, and $84 \%$ participants thought they learned something in the analysis phase.

Scope of observation. Six participants said that shared tags influenced the scope of their observation. One participant told that taking a look at others' tags broadens the scope of observation, and that "If I cannot see the tags other than mine, it will be my one-man play." In contrast, there are opinions that shared tags can narrow a participant's view. For example, participants might only take pictures of the things that are relevant to the previously defined shared tags and overlook things outside the scope of existing tags. A number of comments suggest that determining common tags among the team members during the planning step resulted in negative experiences, making them feel like they are restricted by the previously determined tags. One of the participants felt that such tags were like checklist items that one must follow blindly. He said, "Because it seems that they are created in advance to be like checklist items, you know, so, it was not like attaching tags but more like going OK, check, and done." Another participant discussed the importance of observing the field before determining tags. She said, "If [you] prefer to see the whole thing, [it's better to] look, look and look, on and on and on, really freely without determining, and tags can be inputted while doing that." Coming back to our quantitative results with Figure 3, such restrictive common tags were frequently used during fieldwork in P1F0 and P1F1 conditions. Figure 4 shows that a smaller number of less useful ideas were generated for these conditions. In contrast, private tags were frequently used during fieldwork in $\mathrm{P} 0 \mathrm{~F} 0$ and $\mathrm{P} 0 \mathrm{~F} 1$ conditions. For these conditions, a larger number of more useful ideas were generated. It seems that the discussions to agree on common tags in the planning step were more about compromise than creative fusion, and generated 'checklists' that participants felt obliged to follow. In fact, conventional mobile crowdsourcing with pre-specified tasks closely resemble the environment in which participants simply follow prespecified checklists. Figure 4 then suggest that our method can generate a larger number of useful ideas than the conventional mobile crowdsourcing approach if it is used in appropriate conditions such as P0F0 and P0F1. 
Avoidance of redundancy. Five participants thought that redundancy of collected data is a risk that should be avoided. They also thought that it was 470 possible to avoid redundancy by using shared tags. They pointed out that redundant photos and tags could cause problems because the redundancy could decrease the total amount of information that a team could collect, and duplicate tags and similar tags could cause confusion.

Sense of togetherness. Eight participants said that sharing tags created a sense

475 of togetherness. Some of them also said that such a sense facilitated the mobile work. A frequently updated tag list suggests that the team members are actively making a progress, which can influence a sense of togetherness. Also, tags such as 'cold' can invoke an empathetic connection with team members. Indeed, someone attempted to appropriate the social tagging as a Twitter-like 480 communication tool. Moreover, there was a participant who tagged photos with emoticons.

Sense of Responsibility. Participants said that "When I realized that someone created a lot of tags, I felt that I had to create many tags too" and "this is not competition, but I feel compelled to contribute." Also, when no tags were shared, participants felt that "Since there are no incoming opinions, I felt compelled to find [data] by myself" and "it does not increase unless I input by myself."

\section{Discussion}

\subsection{Mobile Crowdsourcing as Networked Activity}

490 The results of our field trials of co-located mobile collaboration tools suggest that connecting mobile workers creates a network of activities, which could achieve the kind of results that cannot be attained by isolated collection of individuals. In particular, networked activities connects different perspectives and experiences, which can be extremely useful for carrying out tasks that require exploration and creativity, such as mobile crowdsourcing tasks for coping with problems without easy solutions $[10,11,13]$.

This is not to say that mobile workers must always be connected. Rather, we argue that the right blending of private and connected moments needs to be considered in the design of mobile work environments that include not only 500 work itself but also relevant activities such as planning and analysis. The results of our field trials suggest that private and shared tags can be created and used at different phases of mobile work, and the context of creating and using private 
and shared tags influences participants' uses of tags in mobile data collection as we saw in Figure 3

We see that private fieldwork-step tags (solid red) were used 23 percent of the time for P1F0 but only 4 percent for P0F0 in Figure 3. People create and use more private tags in situ when they use common prepared tags, which suggests that people need some private workspaces and that there is some equilibrium point in the continuum between complete connection and separation.

In order to examine the basic impact of collecting data in a group, we chose a small group size with 2-3 members in our field trials. It closely approximates the group sizes in the existing group-based data collection practices which we reported in Section 3.1. We are yet to examine the impact of group sizes. We expect that there can be an optimal group size as Information Foraging theory 515 [27] (p.148) suggests that having more people is not necessarily better.

In our field trials, participants worked together in realtime. However, the proposed workflow does not strictly require synchronous collaboration in each step. What is essential is that participants need to agree on the time to start and finish each step. Therefore, the planning step and the analysis step could be done asynchronously using an online discussion board, and the fieldwork step could also be done asynchronously using a server. As there are pros and cons of asynchronous and synchronous collaboration, we believe it is beneficial that the method itself could accommodate different modes of collaboration.

\subsection{Workflow}

Provision of the right work structures is important for supporting mobile workers. The workflow we used in the field trials is based on the structures of conventional participatory fieldwork events, including the ones we described in Section 3.1. Unlike existing mobile crowdsourcing systems, we consider not only the data collection step but also planning and analysis steps using this workflow. Our survey results suggest that these additional steps are meaningful and can enhance discovery and learning. In particular, the impact of the analysis step on our participants seems very positive.

We also found that the planning step influences the fieldwork step in an interesting way. The tags created in the fieldwork step were used 5 percent of the time for P0F1, but they were used 33 percent of the time for P1F1. This suggests that creating shared tags together in the planning step increases uses of the tags created in the fieldwork step. A possible reason for this is that participants may have established a common ground through the co-creation of tags in the planning step, which possibly made it easier to understand and 
share the tags that others created during the fieldwork step.

\subsection{Mutual Awareness in Distributed Mobile Work}

In Context Weaver, shared tags support awareness about other participants. Participants thought that the tags were generally useful even though they are often very short. The tags are more than just static keywords. They can be interpreted in different ways in different context, and used as sources of awareness and inspiration.

Moreover, shared tags provided social cues for coordination, i.e., the process of managing dependencies among activities [28], such as minimizing redundant work in mobile data collection. This reinforces the discussion that provision of social cues can make information exploration efficient [5]. Also, the tags seemed to influence the motivation of participants who said: "[the tags] saved me from loneliness," "it's good to know what others are doing," or "it's fun to share." The affective aspects of mutual awareness could not be simply overlooked.

Furthermore, our field trials suggested that mutual awareness influenced learning and discovery by participants. Novices could learn from the tags that were created by other participants. Tags may suggest what data other participants are collecting and how they are going about exploring the environment. To realize richer ecologies of participation [29] based on learning and discovery, we need to consider the balance between mutual awareness and private autonomy as we already suggested. If the right balance can be achieved, provision of mutual awareness can be very useful for learning skills and knowledge for and through mobile crowdsourcing.

\subsection{Rapid Feedback}

Our quantitative results show that most participants thought that the analysis step was meaningful and supports discovery and learning. This suggests that it can be worthwhile to incorporate the analysis step in mobile crowdsourcing processes. Rapid feedback is generally useful for making people aware of problems before it's too late. For example, realtime mapping of the overall progress of collaborative citizen sensing can be useful for minimizing redundant work and increasing the spatial coverage quickly. However, in exploratory mobile crowdsourcing, we need more than just monitoring progress. Analyzing and discussing collected data can create a need to collect more data, or to collect data in a different manner. Conventionally, participants do not analyze and discuss field data before they go back to a lab or an office that has the necessary space and tools for analysis. Context Weaver provides support quick in situ analysis based 
on the following two features: (1) shared tagging that allows distributed participants to share analytical insights, and (2) mobile tabletop collaboration that allows participants to organize and analyze photos in face-to-face settings.

Context Weaver's shared tagging provides a generic tool to share keyword tags along with photos. Even though we expected that participants would use this feature to share keywords that simply describe photos, they sometimes used it to share short comments such as "We need more flowers" and "There isn't any place to play basketball," which could be understood as the results of analytical thinking based on what they observed in the field. This suggests an opportunity of a more systematic support to share personal analytical findings and comments easily on a mobile phone, anywhere at anytime.

Discussion Table allows for tabletop collaboration on a mobile device so that participants can analyze and discuss field data face-to-face without going back to a meeting room or a home. In particular, it can facilitate in situ consensus building and group decision making related to data collection. Also, we can use in situ communication to draw out some important information about the field, which resides only in the heads of participants. That said, such a mode of communication would require each team of participants to be co-located and make it difficult to participate when one cannot find co-located peers easily. There is a tradeoff between the rapid in situ feedback that we used in our experiment and ease of participation by a large number of people. Seeking the right mid-point in this tradeoff relationship, we can consider not only participant recruitment but also participant matching to find appropriate peers including friends and strangers.

Oftentimes, photos cannot capture what participants can see, hear, or feel. Still, they are useful as a kind of visual proxies for the actual information, which can help people remember the information, articulate it, and judge its relevance to a current analysis task. It is then extremely important to draw out the information, use it, and record it before participants forget. When participants used Discussion Table in our field studies, they often presented and explained their photos in turn to start a discussion when the images are still 'hot'. Rapid feedback mechanisms in geocentric crowdsourcing should consider an opportunity to use and record information before it is forgotten.

\subsection{Limitations}

610 Our field trials involved 12 and 15 participants to examine usefulness and usability of mobile crowdsourcing tools. Due to the small number of participants as well as the short duration of the experiments, we acknowledge that 
stronger conclusions cannot be made without further experiments with many participants. We compared results in different experimental conditions. However, participants might act differently in authentic environments without the restrictions imposed by the experimental conditions. Other issues may surface, for example, in relation to worker recruitment or reward mechanisms, when the proposed method is evaluated in large-scale crowdsourcing services in the real world. Geocentric mobile crowdsourcing often requires workers to be at relevant locations. An additional constraint is introduced by Context Weaver as it requires one to find co-located peers as well as to be at a relevant location. Although co-located situations can make communication and collaboration easier, there can be cases that finding appropriate co-located peers is not easy. It would be therefore valuable to consider peers who are distributed in time and space.

${ }_{625}$ As we discussed, the proposed method does not strictly require synchronous, real-time collaboration in each of the planning, fieldwork and analysis steps. However, we are yet to examine user experiences that involve asynchronous modes of collaboration.

\section{Conclusion}

We have discussed the design of networked mobile crowdsourcing tools based on the field trials of Context Weaver. In particular, we proposed a method to provide mutual awareness and rapid feedback considering the workflow involving the planning, fieldwork and analysis phases. Although we cannot make strong conclusions based on the field trials with a small number of participants, our results suggest that the proposed method can generate a larger number of useful ideas than the conventional mobile crowdsourcing approach if it is used in appropriate conditions.

Our future work includes a group-based recruitment mechanism considering geographic constraints and social factors. Such a mechanism could exploit self-organizing group formation among friends and strangers as well as simpler task-worker matching. A relevant research question may concern the impact of the characteristics of groups on the quality and reliability of the outcomes of crowdsourced mobile work.

In the future, various mobile crowdsourcing events could be connected across time and space so that people can build on existing results by others, and collectively address difficult challenges. We believe that pervasive networks of people, places, and events could play an important role in bridging different communities and relevant mobile crowdsourcing events. 


\section{Acknowledgement}

This research was supported by the Green Network of Excellence Program of MEXT, Japan and the Promoting Science and Technology System Reform Program of JST, Japan.

\section{References}

[1] FixMyStreet.

URL http://www.fixmystreet.jp/

[2] T. Erickson, Geocentric Crowdsourcing and Smarter Cities: Enabling Urban Intelligence in Cities and Regions, in: First International Workshop on Ubiquitous Crowdsourcing (in conjunction with UbiComp'10 Conference), 2010 .

[3] M. M. Webber, Dilemmas in a General Theory of Planning *, Policy Sciences 4 (1973) (1973) 155-169.

[4] L. A. Suchman, Plans and situated actions: the problem of human-machine communication, Cambridge University Press, 1987.

[5] E. H. Chi, Information Seeking Can Be Social, Computer 42 (3) (2009) 42-46. doi:10.1109/MC.2009.87.

[6] P. Isenberg, D. Fisher, S. a. Paul, M. R. Morris, K. Inkpen, M. Czerwinski, Co-located collaborative visual analytics around a tabletop display., IEEE transactions on visualization and computer graphics 18 (5) (2012) 689-702. doi:10.1109/TVCG.2011.287.

[7] Ushahidi, Ushahidi.

URL http://www.ushahidi.com

[8] A. Manzoor, C. Patsakis, A. Morris, J. McCarthy, G. Mullarkey, H. Pham, S. Clarke, V. Cahill, M. Bouroche, CityWatch: exploiting sensor data to manage cities better, Transactions on Emerging Telecommunications Technologies 25 (1) (2014) 64-80. doi:10.1002/ett. 2786.

[9] E. Aubry, T. Silverston, A. Lahmadi, O. Festor, CrowdOut : a Mobile Crowdsourcing Service for Road Safety in Digital Cities, in: Int'l Workshop on Crowdsensing Methods, Techniques, and Applications, 2014, pp. 86-91. 
[10] D. Christin, C. Roß kopf, M. Hollick, uSafe: A privacy-aware and participative mobile application for citizen safety in urban environments, Pervasive and Mobile Computing 9 (5) (2013) 695-707. doi:10.1016/j.pmcj.2012.08.005.

[11] S. Kim, J. Mankoff, E. Paulos, Sensr: evaluating a flexible framework for authoring mobile data-collection tools for citizen science, in: Proc. CSCW, 2013, pp. 1453-1462.

[12] S. Reddy, D. Estrin, M. Srivastava, Recruitment Framework for Participatory Sensing Data Collections, in: Proc. Pervasive, 2010, pp. 138-155.

[13] W. Willett, P. Aoki, N. Kumar, S. Subramanian, A. Woodruff, Common sense community: scaffolding mobile sensing and analysis for novice users, in: Proc. Pervasive, 2010, pp. 301-318.

[14] F. Alt, A. S. Shirazi, A. Schmidt, U. Kramer, Z. Nawaz, Location-based Crowdsourcing : Extending Crowdsourcing to the Real World, in: Proc. NordiCHI, 2010, pp. 13-22.

[15] Y. Pan, E. Blevis, A Survey of Crowdsourcing as a Means of Collaboration and the Implications of Crowdsourcing for Interaction Design, in: Proc. 2011 Int. Conf. on Collaboration Technologies and Systems (CTS), Ieee, 2011, pp. 397-403. doi:10.1109/CTS.2011.5928716.

[16] A. Kittur, B. Smus, S. Khamkar, R. Kraut, CrowdForge : Crowdsourcing Complex Work, in: Proc. UIST, 2011, pp. 43-52.

[17] T. Buecheler, J. H. Sieg, R. M. Füchslin, R. Pfeifer, Crowdsourcing, Open Innovation and Collective Intelligence in the Scientific Method : A Research Agenda and Operational Framework Why Crowdsourcing in the Scientific Method, in: Proc. Alife XII Conference, 2010, pp. 679-686.

[18] A. Kittur, R. E. Kraut, Harnessing the Wisdom of Crowds in Wikipedia: Quality Through Coordination, in: Proc. CSCW, 2008, pp. 37-46.

[19] H. Väätäjä, P. Egglestone, Briefing News Reporting with Mobile Assignments Perceptions, Needs and Challenges, in: Proc. CSCW, 2012, pp. 485-494.

[20] J. Fails, K. Herbert, E. Hill, C. Loeschorn, S. Kordecki, D. Dymko, A. DeStefano, Z. Christina, GeoTagger : A Collaborative and Participatory Environmental Inquiry System, in: CSCW Companion, 2014, pp. 157-160. 
[21] M. L. Maher, J. Preece, T. Yeh, C. Boston, K. Grace, A. Pasupuleti, A. Stangl, NatureNet: A Model for Crowdsourcing the Design of Citizen Science Systems, in: CSCW Companion, 2014, pp. 201-204.

[22] J. P. Spradley, Participant observation, Harcourt Brace Jovanovich College Pbulishers, New York, 1980.

[23] P. Pirolli, S. Card, Information foraging, Psychological review 106 (4) (1999) 643-675.

[24] H. Sasao, T., Konomi, S., Arikawa, M. and Fujita, Poster Abstract: Context Weaver: Linking Qualitative Spatial Information for Community Mapping, in: Asia Pacific Conf. on Computer Human Interaction (APCHI), 2012.

[25] D. E. Difallah, G. Demartini, P. Cudré-mauroux, Pick-A-Crowd : Tell Me What You Like, and I 11 Tell You What to Do, in: Proceedings of 725 WWW'13, 2013, pp. 367-374.

[26] M.-C. Yuen, I. King, K.-S. Leung, TaskRec: A Task Recommendation Framework in Crowdsourcing Systems, Neural Processing Letters II (2014) 516-525. doi:10.1007/s11063-014-9343-z.

[27] P. Pirolli, Information Foraging Theory, Oxford University Press, 2007.

[28] T. W. Malone, K. Crowston, The Interdisciplinary Study of Coordination, ACM Computing Surveys 26 (1) (1994) 87-119. doi:http://doi.acm.org/10.1145/174666.174668.

[29] G. Fischer, Understanding, Fostering, and Supporting Cultures of Participation, interactions 18 (3) (2011) 42-53. doi:10.1145/1962438.1962450. 
Ms. Sasao Tomoyo is a graduate student at the University of Tokyo.

Dr. Shin'ichi Konomi is an associate professor of the Center for Spatial Information Science (CSIS), the University of Tokyo. His research interests include ubiquitous computing (UBICOMP), human-computer interaction (HCI), context-awareness, urban computing, radio frequency identification (RFID), technology and privacy, and computer-supported cooperative work (CSCW). He received his Ph.D. in computer science from Kyoto University. More info at: http://www.csis.u-tokyo.ac.jp/ konomi.

Dr. Masatoshi Arikawa is a professor of the Center for Spatial Information Science (CSIS), the University of Tokyo.

Dr. Hideyuki Fujita is an assistant professor at University of Electro-Communications. 


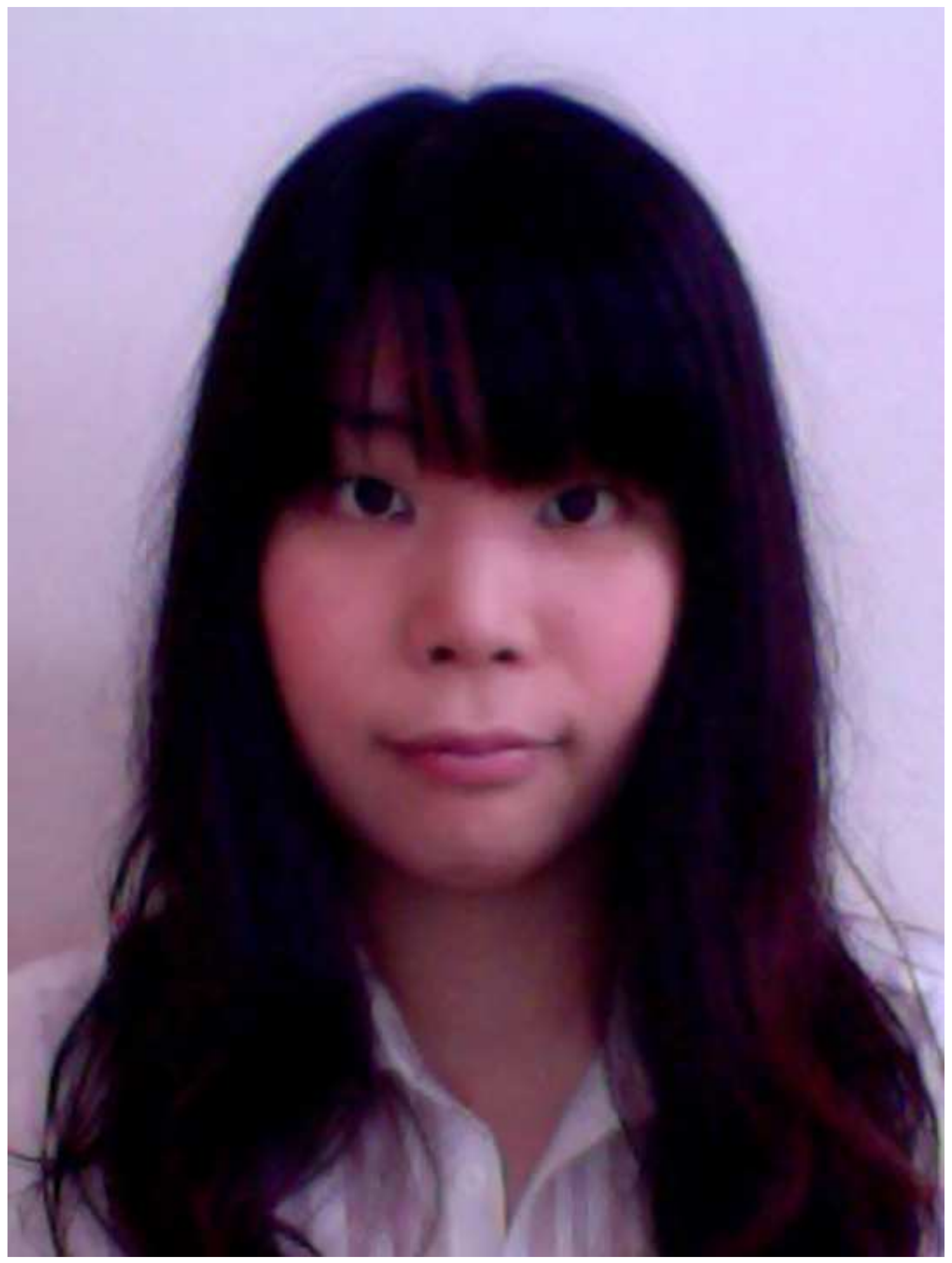




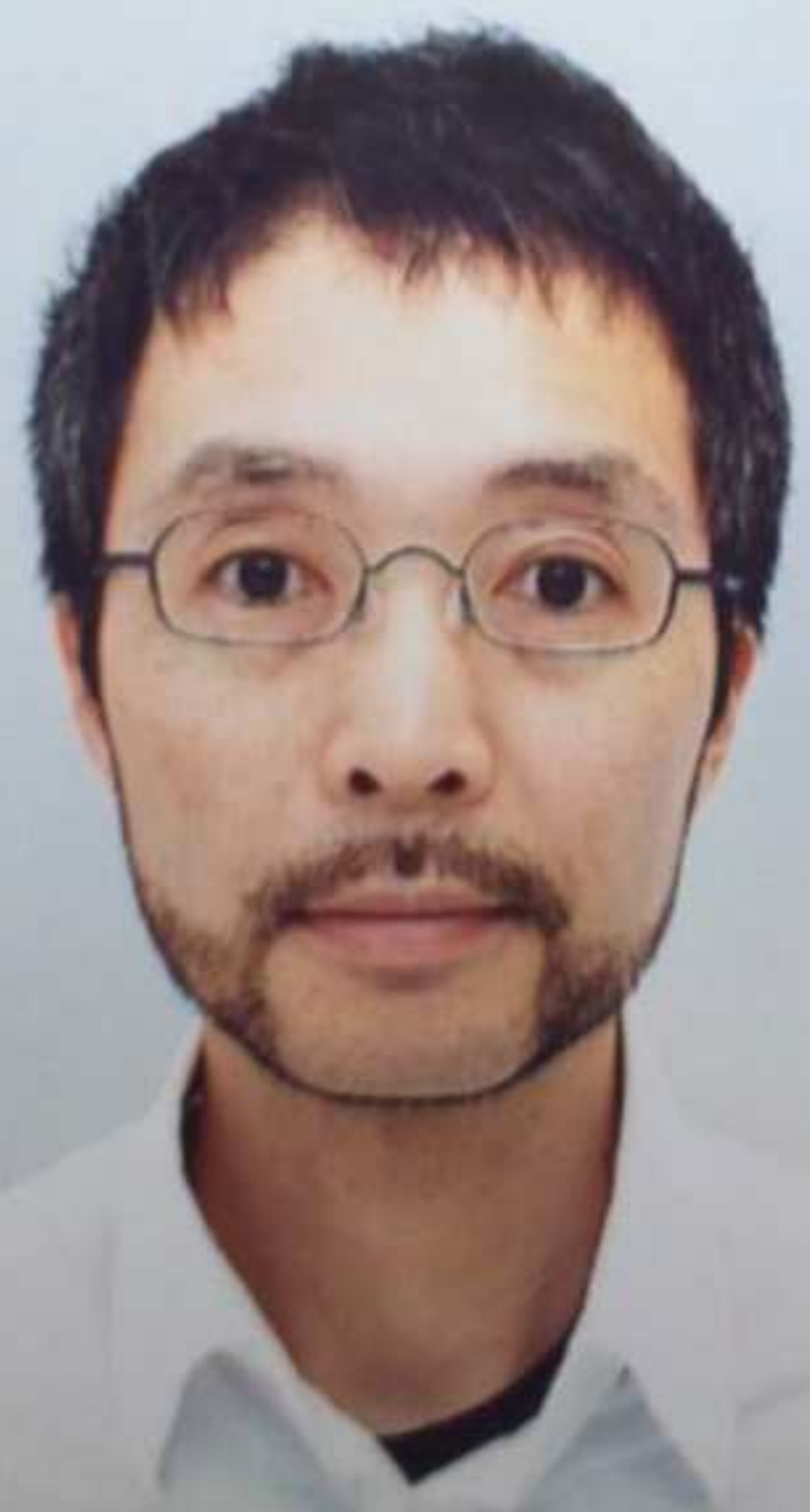




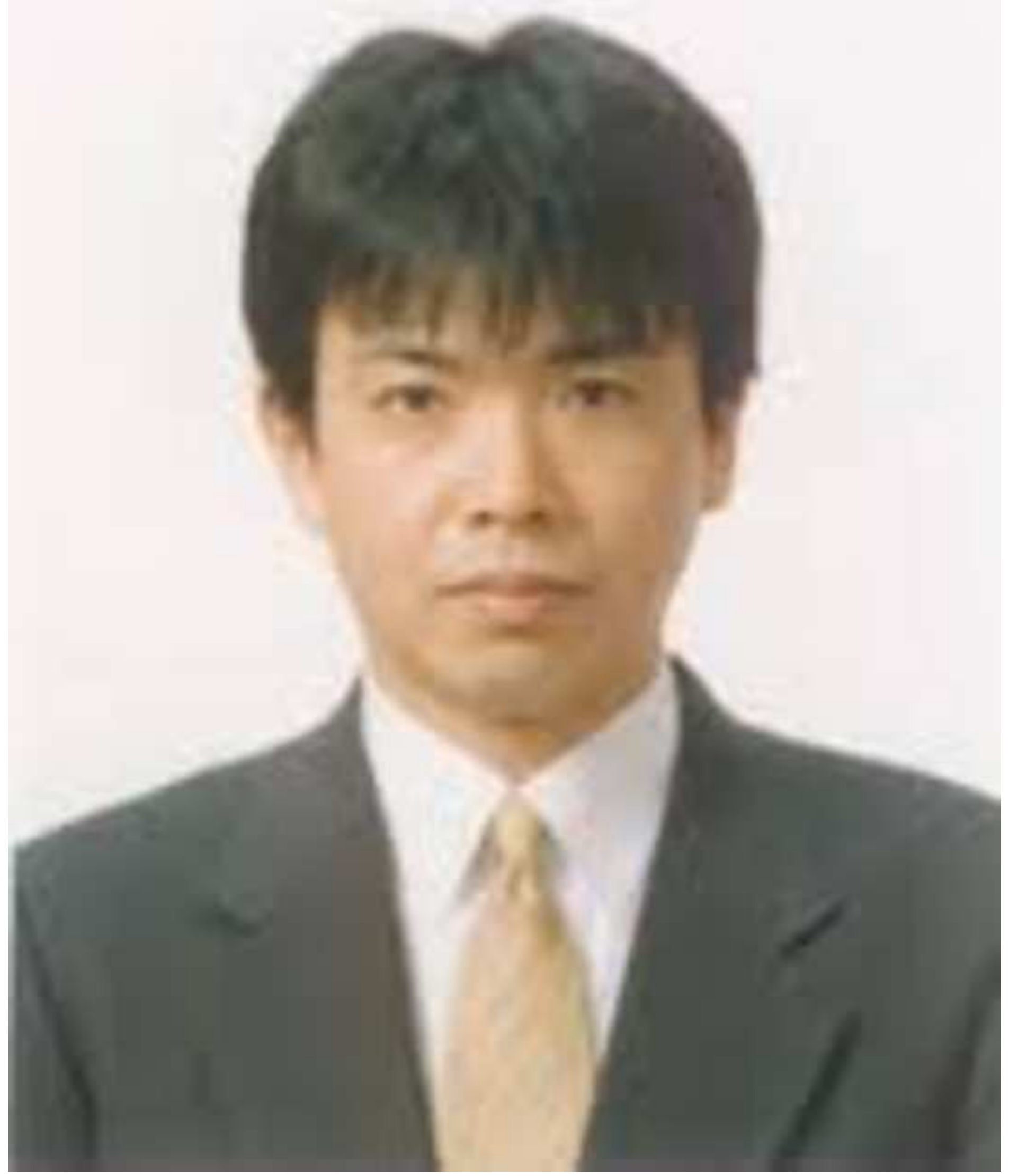




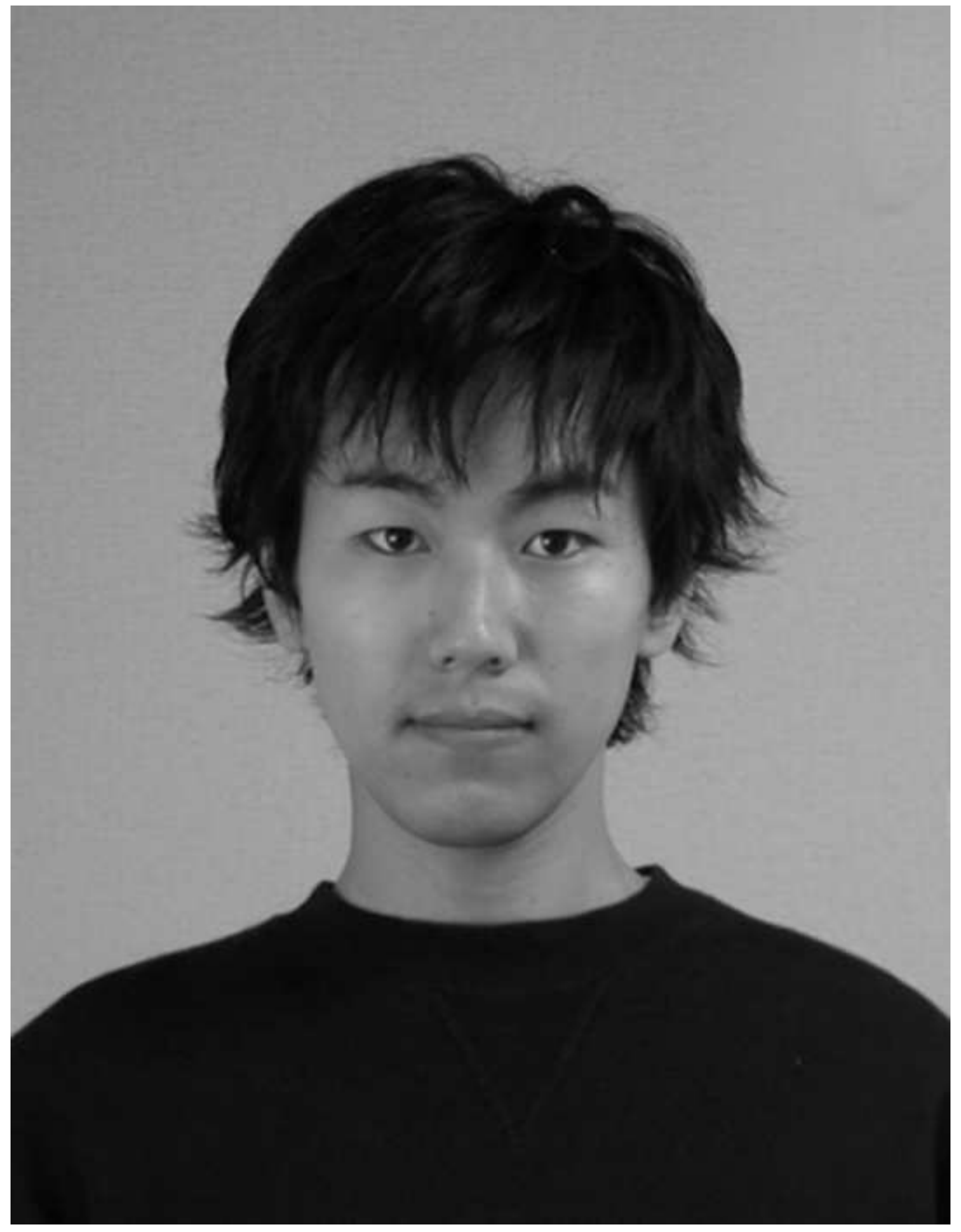

